\title{
Phenylboronic acid-decorated polymeric nanomaterials for advanced bio-application
}

https://doi.org/10.1515/ntrev-2019-0049

Received Apr 22, 2019; accepted Aug 22, 2019

\begin{abstract}
The paradigm of using phenylboronic aciddecorated polymeric nanomaterials for advanced bioapplication has been well established over the past decade. Phenylboronic acid and its derivatives are known to form reversible complexes with polyols, including sugar, diol and diphenol. This unique chemistry of phenylboronic acid has given many chances to be exploited for diagnostic and therapeutic applications. This review highlights the recent advances in fabrication of phenylboronic aciddecorated polymeric nanomaterials, especially focus on the interactions with glucose and sialic acid. Applications of these phenylboronic acid-decorated nanomaterials in drug delivery systems and biosensors are discussed.
\end{abstract}

Keywords: Phenylboronic acid; Polymeric nanomaterials; Polyols; Drug delivery

\section{Introduction}

Phenylboronic acid (PBA) and its derivatives are known to reversibly form cyclic boronic esters with polyol compounds $[1,2]$, hence they could recognize carbohydrates, such as glucose in blood and sialic acid (SA) on cancer cells. Carbohydrates are the foundation of life and play irreplaceable roles in a variety of cellular events, including proliferation, recognition, intercellular communication and immunoregulation. When disease occurs, the type and expression amount of carbohydrates would change. The unique chemistry of PBA with polyol compounds, especially carbohydrates, provides a way to de-

\footnotetext{
${ }^{\star}$ Corresponding Author: Qianqian Guo: Department of pharmaceutical Engineering (State Key Laboratory of Functions and Applications of Medicinal Plants, the Key Laboratory of Optimal Utilization of Natural Medicine Resources), School of Pharmaceutical Sciences, Guizhou Medical University, Guiyang, Guizhou 550025, China; Email: guoqqsci@126.com

Tianyu Lan: School of Chemical Engineering, Guizhou Minzu University, Guiyang 550025, Guizhou Province, China
}

sign PBA-based polymeric nanomaterials. As polymeric nanomaterials could greatly enhance molecular interactions by multivalent effects, they have attracted much attention in biomedical applications [3-5], such as drug delivery systems and biosensors for diagnosis and therapeutics of diseases [6].

The most typical application of PBA and its derivatives is focused on glucose-responsive materials to recognize blood glucose and delivery insulin for the treatment of diabetes mellitus [7-10]. However, PBA-based glucoseresponsive materials could only work in alkaline media but not at physiological $\mathrm{pH}$, due to the high $\mathrm{p} K_{a}$ value ( $\mathrm{p} K_{a}$ > 8.0) of PBA moiety [11-14]. Numerous investigations have been focused on the design and synthesis of PBA-based polymers to decrease $\mathrm{p} K_{a}$ of PBA moiety and increase the glucose-sensitivity as well as effectively release insulin at physiological pH [15-18]. Interestingly, PBA and its derivatives can stably bind with SA in acid conditions, such as tumor acid microenvironment, resulting in another application of PBA-based polymeric nanomaterials to construct anticancer drugs delivery systems [19-21].

Herein, we review the recent advances in fabrication of PBA-decorated polymeric nanomaterials based on the specific interactions with carbohydrates, and highlight the biomedical applications in drug delivery systems and biosensors.

\section{PBA-based glucose-sensitive polymeric nanomaterials}

Insulin-dependent diabetes mellitus characterized by accumulating glucose concentrations in blood has been a major global health epidemic [22, 23], leading to a highly need to design glucose-responsive materials for self-regulated insulin release responding to the variation of blood glucose concentrations. PBA-based glucose-responsive polymeric materials have been wellinvestigated because they are versatile enough to design different formulations and more stable than proteinbased systems (glucose oxidase and concanavalin A)

¿ Open Access. (c) 2019 T. Lan and Q. Guo, published by De Gruyter. (Cc) BY 4.0 License 
[24]. The interaction between PBA and glucose is reversible equilibrium, which lays the foundation for the glucose-responsiveness of PBA-based materials. PBAbased glucose-sensitive nanomaterials could be fabricated by different approaches, such as self-assembly of amphiphilic copolymers, polymerizing into nano/microgels, and conjugating with inorganic hybrid nanoparticles, etc. [25-31].

As most of the PBA-based materials reported demonstrate maximum glucose-sensitivity at $\mathrm{pH} 9 \sim 10$, significant efforts have been focused on decreasing their $\mathrm{p} K_{a}$ values and enhancing the sensitivity to glucose at physiological conditions. Sumerlin group prepared macromolecular stars with boronic ester linkages to self-assemble into nanoparticles [25]. The boronic esters were formed between 3-acrylamidophenyl boronic acid (APBA) and monofunctional diols, and the formation-dissociation process was proved to be repeatable over multiple cycles (Figure 1A). Later, they synthesized another boronic acidbased polymeric aggregations with glucose-sensitivity at $\mathrm{pH}=7.4$, indicating promising prospects for controlled insulin delivery systems under physiological conditions (Figure 1B) [26]. Kim group prepared two kinds of insulin-loaded polymersomes from polyboroxole-based block copolymers, which could release insulin responding to the glucose-triggered disassembly under physiologically conditions (see Figure 2) [28, 29]. Differently, Yao et al. designed a novel nanocarrier with phenylboronate ester as a leaving group in the hydrophobic block, and the nanocarrier successfully realized glucose-responsiveness and insulin release at neutral pH (Figure 3) [27].

In recent years, Zhang group synthesized a series of PBA-based amphiphilic copolymers by radical polymerization and living/controlled polymerization to delivery insulin at physiological conditions [32-37]. By introducing carbohydrate moieties into copolymers, $\mathrm{p} K_{a}$ values of PBA moieties were decreased and glucose sensitivity was enhanced at physiological conditions, which ensured the delivery effectiveness and self-regulated release of insulin. To explore the effect of polymer structures on self-assembled aggregates and glucose-sensitivity, a series of amphiphilic block and random PBA-based copolymers with similar monomer compositions were synthesized by reversible addition-fragmentation chain transfer (RAFT) polymerization [38]. These copolymers could easily self-assemble into spherically shaped nanoparticles in aqueous solution and two types of nanoparticles facilitated insulin delivery at physiological $\mathrm{pH}$, despite insulin release rate of random copolymer was slightly quicker than that of the block ones (Figure 4).

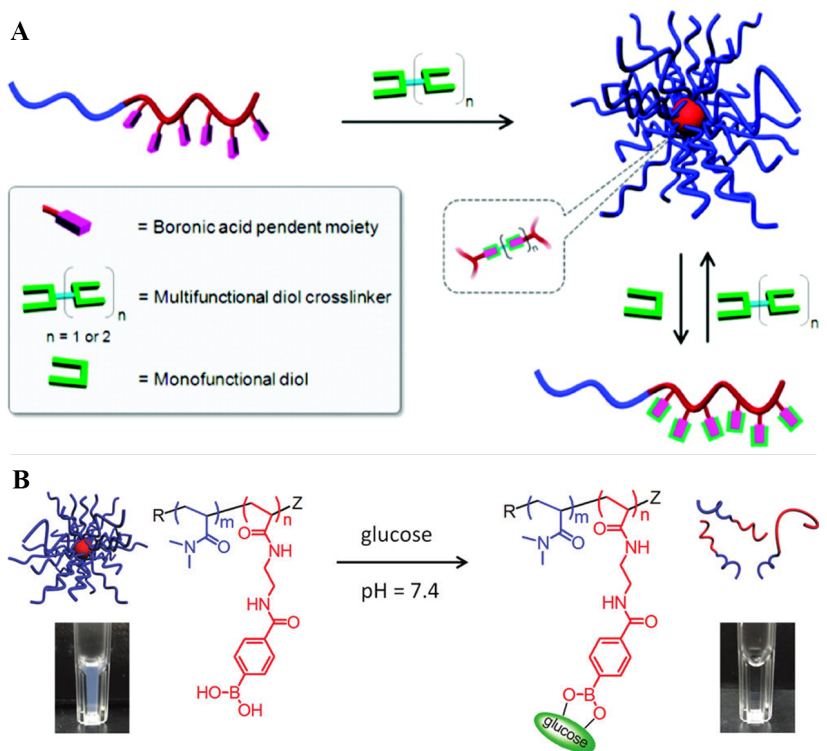

Figure 1: (A) Formation and disintegration of dynamic-covalent nanoaggregates via boronic ester linkages. Reprinted with permission from [25]. Copyright 2011, American Chemical Society. (B) Schematic illustration for glucose-triggered dissociation of the nanoparticles. Reprinted with permission from [26]. Copyright 2012, American Chemical Society

Besides PBA-based amphiphilic copolymers selfassembling into nanoparticles, the chemically crosslinked PBA-decorated nanogels (microgels) also attract much attention because they can meet different needs, such as prompt drug delivery, oral administration, and intravenous injection. Typical PBA-based glucoseresponsive nanogels were prepared through modification of poly $(N$-isopropylacrylamide-co-acrylic acid) [P(NIPAMco-AA)] with 3-amino-phenylboronic acid (Figure 5) [3941]. The introduction of PBA groups significantly decreased volume phase transition temperature of microgels, yet a pH value higher than 7.4 was needed to release loaded-insulin in response to glucose.

Wang et al. reported multifunctional microgels based on PNIPAM, $N$-acryloyl-3-aminophenylboronic acid and two fluorescent molecules [42]. The FRET efficiencies and shrink-swelling properties of microgels could be tuned via thermo-induced collapse or glucose-induced swelling at proper $\mathrm{pH}$ and temperature (Figure 6). Tang et al. synthesized a specific glucose-sensitive P(NIPAM-AAPBA) microgel that displayed specific glucose sensitivity at physiological pH [43]. Due to the interference from fructose, galactose, and lactate was negligible, the author considered the contraction-type specific glucose-sensitive microgels had potential applications in self-regulated insulin delivery and glucose sensing (Figure 7). Zhao et al. prepared a novel nanogel with high glucose sensitivity 
A
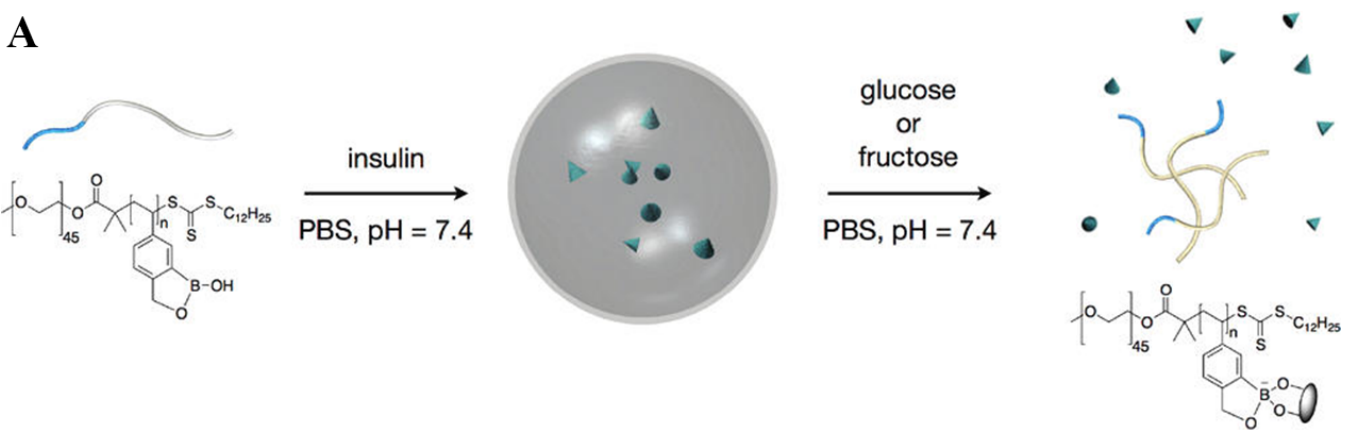

B
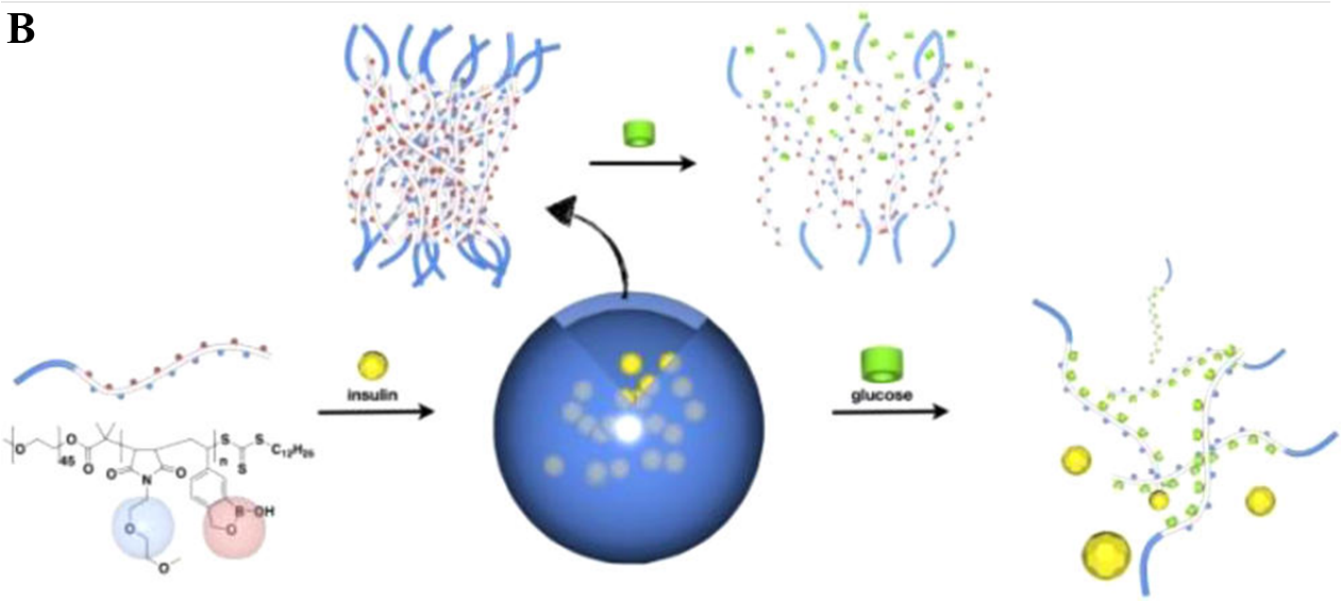

Figure 2: (A) Self-assembly of PEG-b-PBOx polymersome and its disassembly responding to monosaccharides. Reprinted with permission from [28]. Copyright 2012, American Chemical Society. (B) Schematic illustration for self-assembly and glucose-responsive disassembly of polymersomes. Reprinted with permission from [29]. Copyright 2012, American Chemical Society

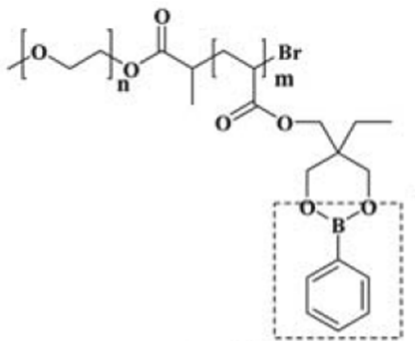

Amphiphilic polymer

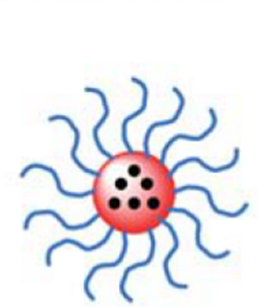

Micellar aggregates
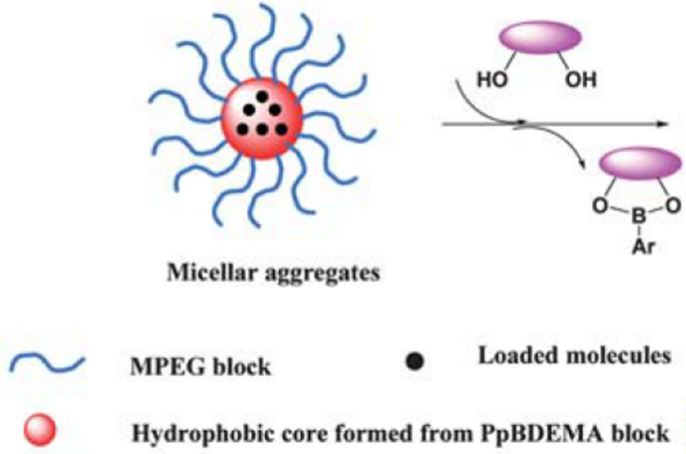

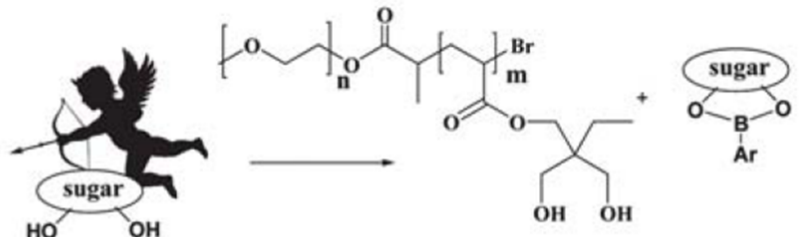

Double hydrophilic polymer

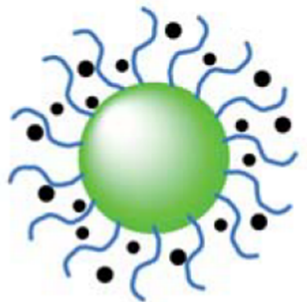

$\mathrm{HO}_{\mathrm{OH}}$ Sugar molecule

Swollen core after sugar-responsiveness

Figure 3: Schematic illustration for sugar-responsive behavior of nanoassembly. Reproduced with permission from [27]. Copyright 2011, The Royal Society of Chemistry 

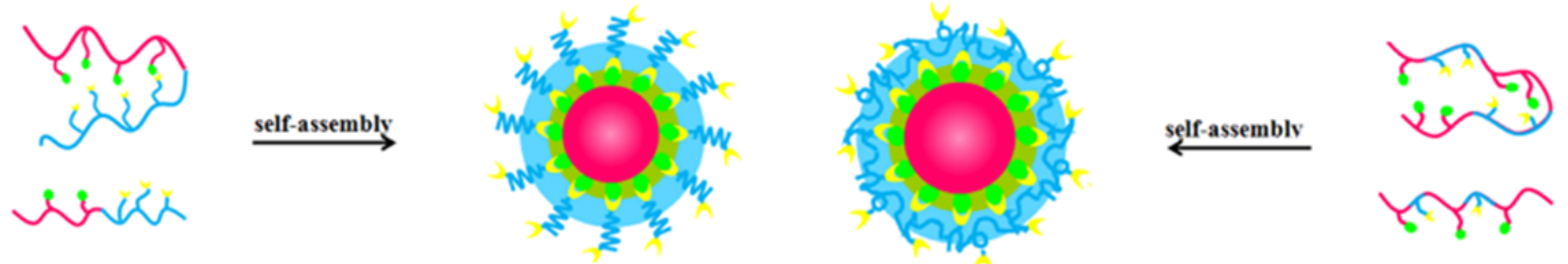

\section{p(AAPBA- $b$-AGA)}

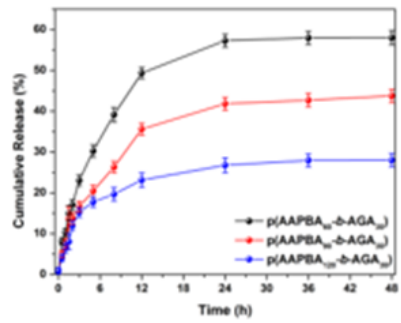

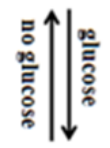

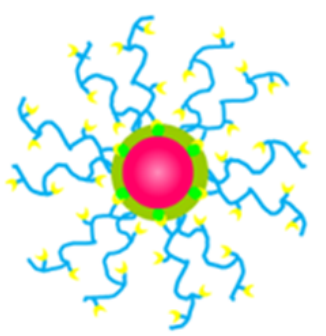

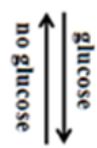

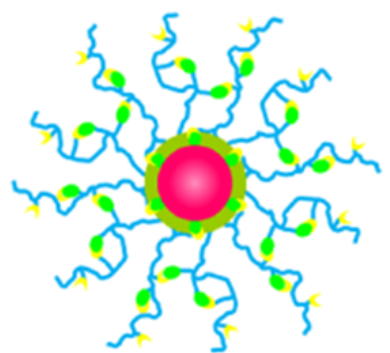

p(AAPBA-r-AGA)

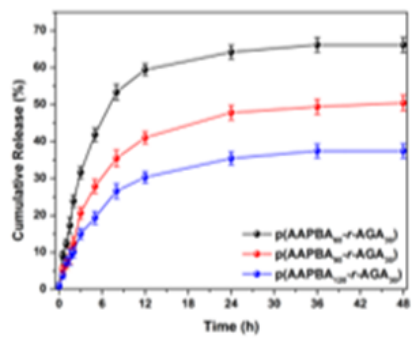

Figure 4: Schematic illustration for the self-assembly and insulin release behaviors of block and random copolymeric nanoparticles. Adapted with permission from [38]. Copyright 2015, American Chemical Society

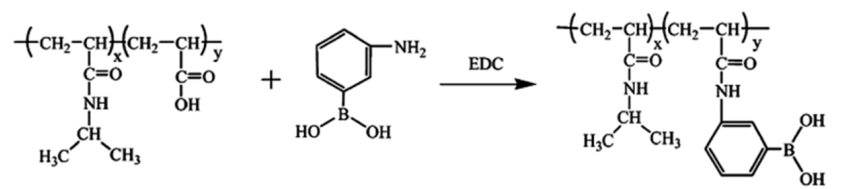

Figure 5: Synthesis of glucose-sensitive P(NIPAM-PBA) microgels with pendant 3-amino-phenylboronic acid. Adapted with permission from [41]. Copyright 2006, American Chemical Society
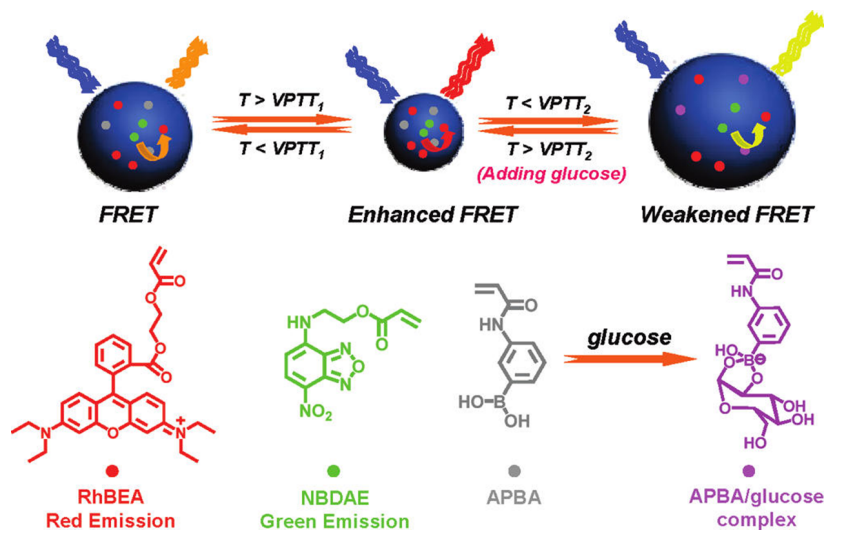

Figure 6: Synthesis and modulation of FRET efficiencies for microgels by temperature variations and adding glucose. Reprinted with permission from [42]. Copyright 2011, American Chemical Society

through one-pot thiol-ene copolymerization of pentaerythritol tetra(3-mercaptopropionate), poly(ethylene glycol) diacrylate, methoxyl poly(ethylene glycol) acrylate and $N$ acryloyl-3-aminophenylboronic acid [44]. The release of loaded-insulin was triggered and modulated at $\mathrm{pH} 7.4$ responding to different glucose concentrations (Figure 8).

To simultaneously achieve optical detection of glucose, high drug loading capacity, and self-regulated drug delivery, $\mathrm{Wu}$ and coauthors reported a series of PBA-based hybrid microgels with glucose-sensitivity to realize self-regulated insulin delivery at physiological $\mathrm{pH}$ [16]. The multifunctional hybrid nanogels were made of Ag nanoparticle cores covered with a copolymer gel shell of poly(4-vinylphenylboronicacid-co-2(dimethylamino)ethylacrylate) [p(VPBA-DMAEA)] [16, 4547]. The small sized Ag cores $(10 \pm 3 \mathrm{~nm})$ in microgels provided fluorescence as an optical code. The microgels could not only convert the disruption in homeostasis of glucose level into optical signals to detect the blood glucose levels, but also regulate release of preloaded insulin responding to the glucose level variations (Figure 9A). Later, they reported a fluorescent responsive hybrid nanogel of Zinc oxide@poly[ $N$-isopropylacrylamide (NIPAM)-acrylamide (AAm)-2-aminomethyl-5-fluorophenylboronic acid (FPBA)] [45]. The hybrid nanogels could sensitively and selectively detect glucose via fluorescent signals over the clinically relevant glucose concentrations of $18-540 \mathrm{mg} / \mathrm{dl}$. The nanogels could also regulate loaded-insulin release behaviors in response to different glucose levels and exhibit quicker release rate at higher glucose level (Figure 9B). 

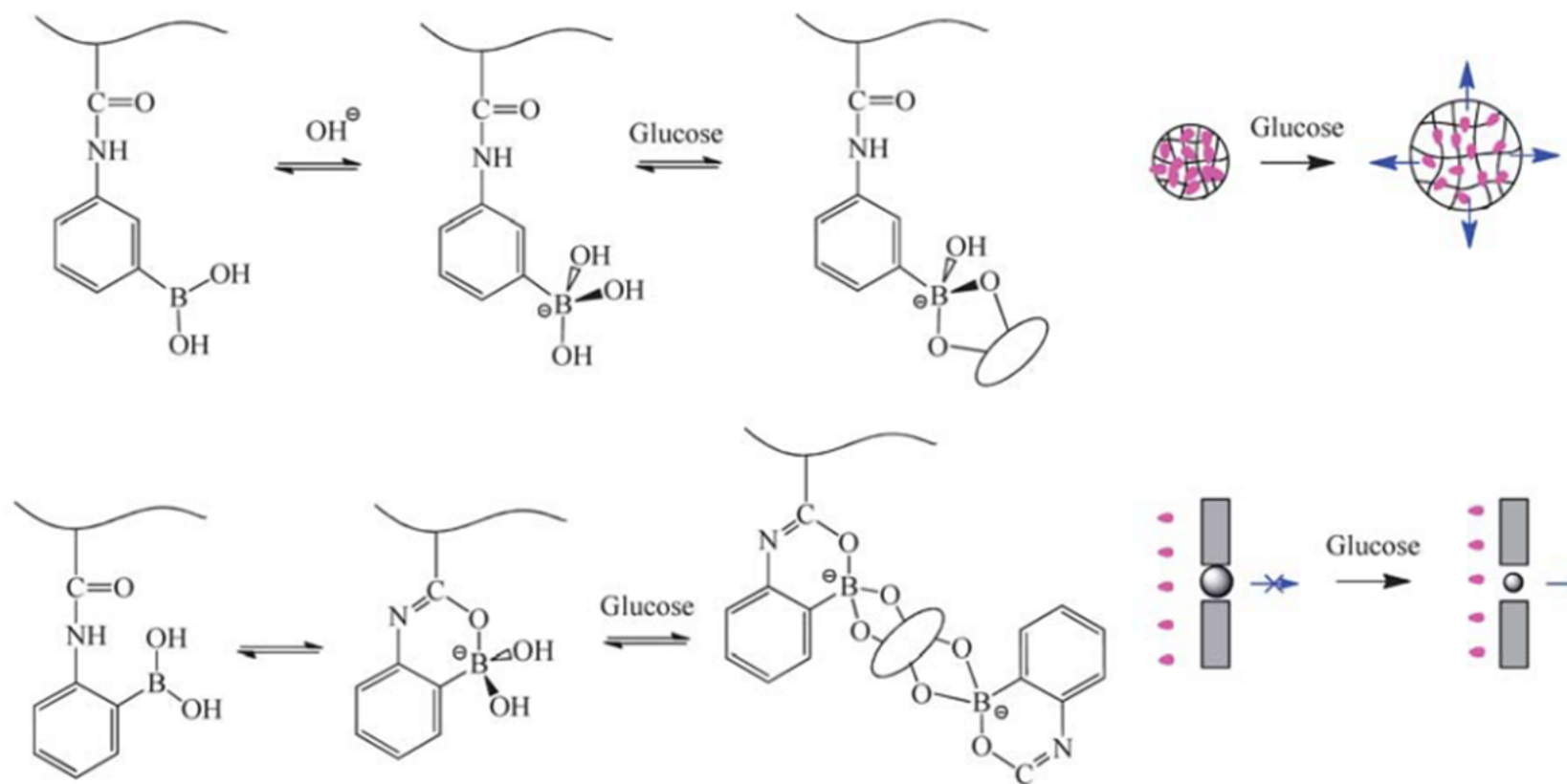

Glucose
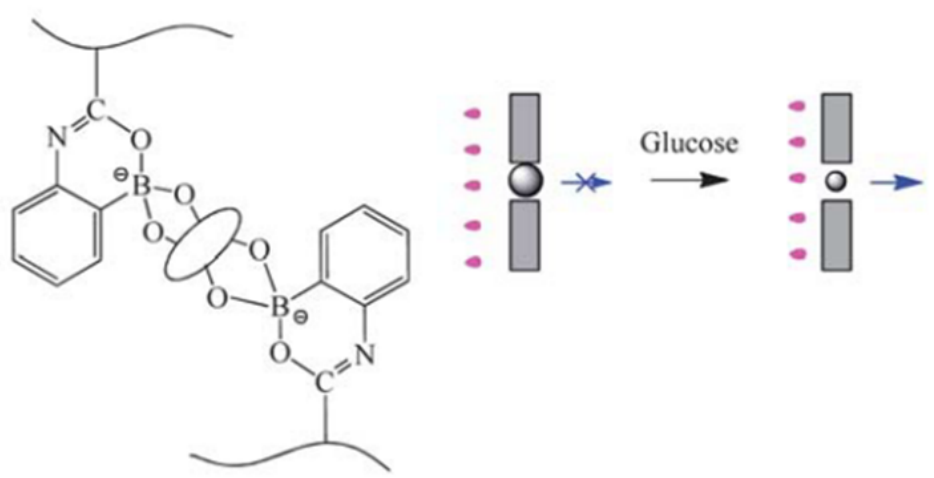

Figure 7: The complexation equilibrium between glucose and PBA groups, and the glucose-induced swelling of P(NIPAM-AAPBA) microgels. Reproduced with permission from [43]. Copyright 2014, The Royal Society of Chemistry

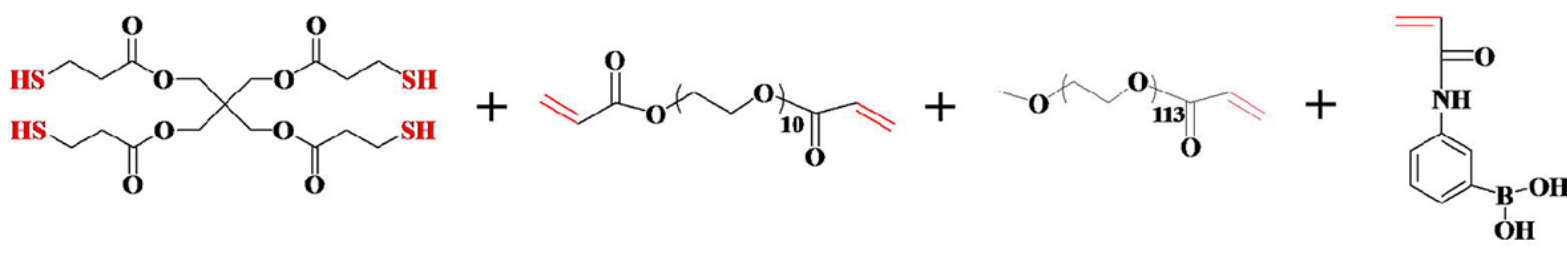

$\mathbf{O} \equiv \mathbf{Q T}$

$m=$ PEGDA

min $\equiv$ PEGA $\quad \downarrow \equiv$ AAPBA

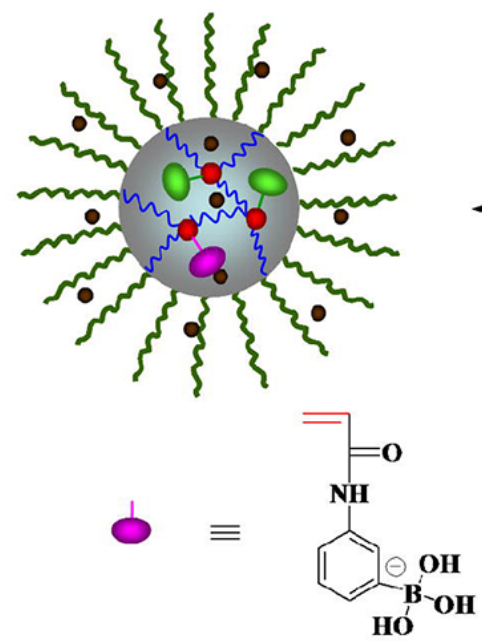

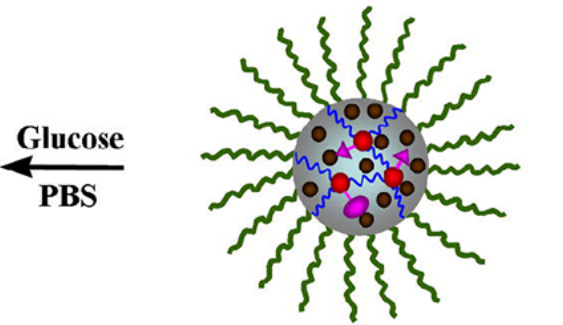

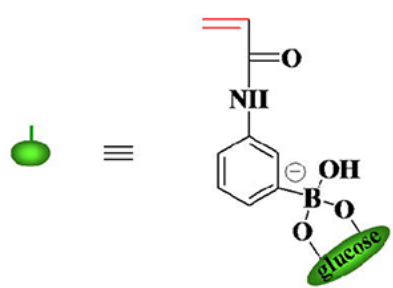

ARS

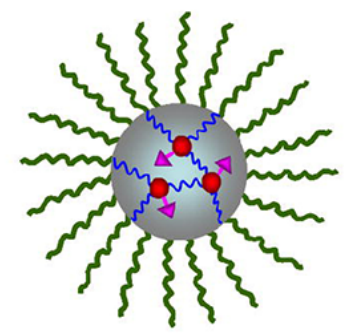

$\mathrm{O} \equiv \mathbf{A R S}$

Figure 8: Structures of nanogels and glucose-sensitive behaviors of ARS-loaded nanogels in PBS [44]. Reprinted with permission from [44]. Copyright 2013, Elsevier 


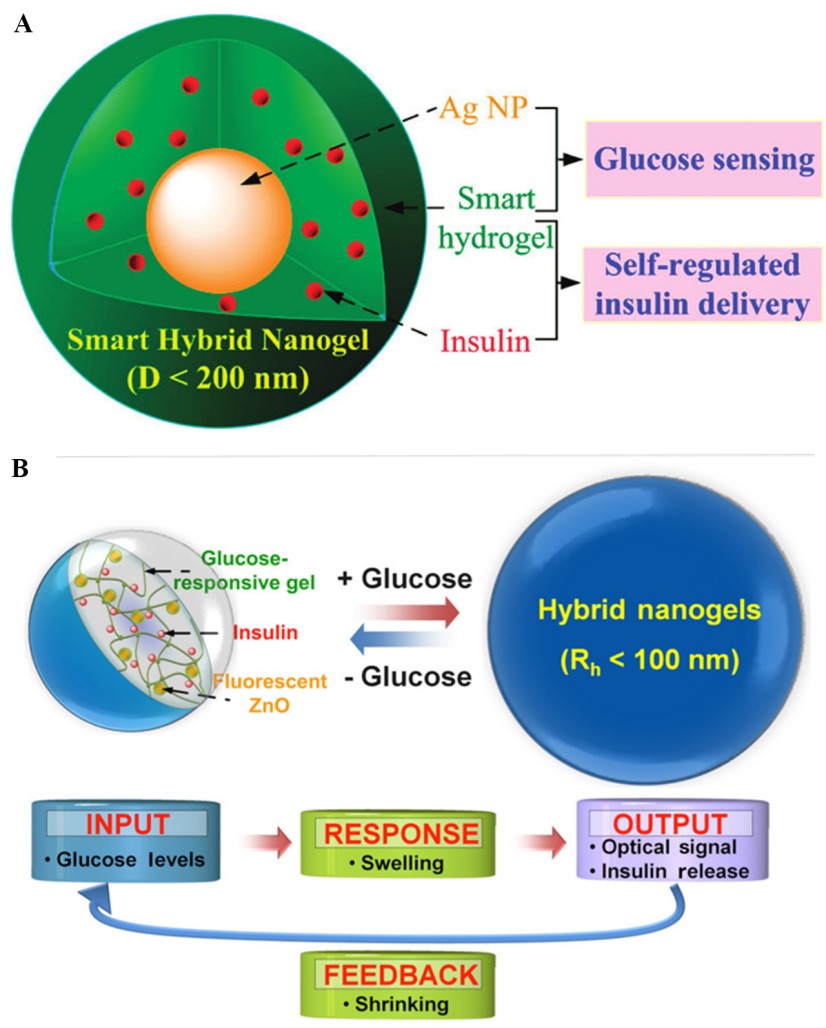

Figure 9: (A) Smart hybrid nanogels for integration of optical glucose detection and self-regulated insulin delivery into a single nano-object. Reprinted with permission from [16]. Copyright 2010 American Chemical Society. (B) A single hybrid nanogel for integration of fluorescent glucose sensing and insulin delivery. Reprinted with permission from [45]. Copyright 2012, SAGE Publications

\section{PBA-mediated SA recognition on cancer cells}

SA is an anionic monosaccharide that frequently occurs at the termini of glycan chains. Its overexpression on cell membranes implicated in the malignant and metastatic phenotypes of various types of cancers, such as lung, breast, colon, prostate, bladder, and stomach cancer [48, 49]. Thus, advanced techniques to monitor SA expression would have great significance on diagnosis and therapeutics. Interestingly, PBA and its derivatives can recognize SA and form the stable boronic ester with SA not only in the physiological and basic $\mathrm{pH}$ but even in acidic medium such as tumor acidic microenvironment [50, 51]. Thus, many researchers have devoted to explore the advanced materials based on PBAmediated SA-recognition as cancer diagnostic and therapeutic agents [52-58]. Matsumoto et al. prepared a PBA modified monolayer gold electrode to noninvasively detect SA on cell membranes [52]. Deng et al. prepared glucose-

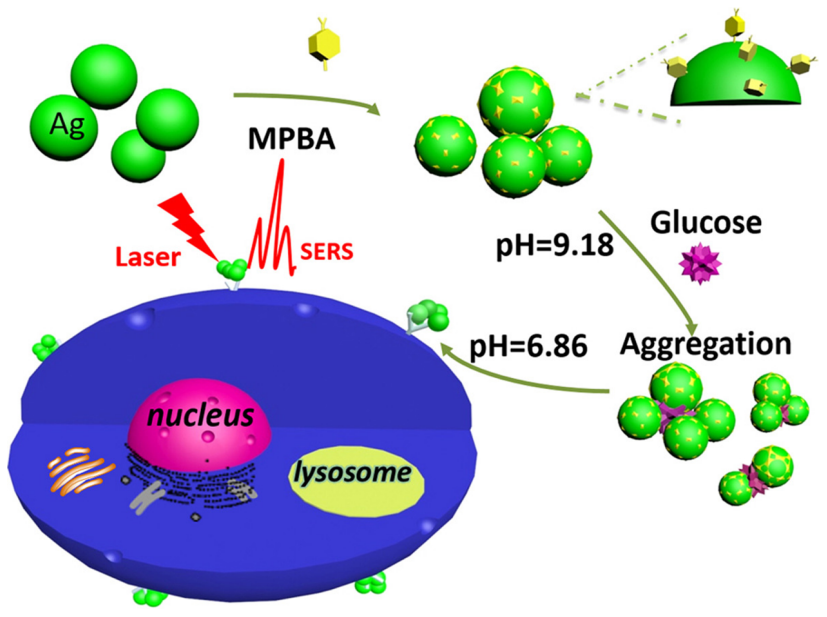

Figure 10: Targeted identification of the cancer cell by a highly sensitive Glucose-MPBA@AgNPs nanoprobes. Reprinted with permission from [54]. Copyright 2017, Elsevier

bridged and 4-mercaptophenyl boronic acid-decorated silver nanoparticle (Glucose-MPBA@AgNPs) as nanoprobe for highly sensitive recognition of SA expression levels on the surfaces of both normal and cancer cells via surfaceenhanced raman scattering spectroscopy (Figure 10) [54].

Among these materials, polymeric nanoparticles occupy the dominant roles owing to their excellent expression in the cancer diagnostic and therapeutic fields [5963]. Liu et al. prepared PBA and amphiphilic copolymer of maleic anhydride/octadecene coated with semiconductor quantum dots as imaging probes to specifically label SA on living cells [62]. These probes could one-step label and continuously track SA moieties on cell surfaces without any pretreatment of living cells (Figure 11). Zhang et al. prepared a SA-recognition system based on gold nanoparticles (AuNPs) labeled on biotinylated phenylboronic acid (biotin-APBA), in which AuNPs enabled to enhance signal in an inductively coupled plasma mass spectrometry [63]. By taking HepG2 and MCF-7 cells as model cancer cells, the average numbers of SA expressed on the single MCF-7 and HepG2 cell surface were $7.0 \times 10^{9}$ and $5.4 \times 10^{9}$, respectively. The method is helpful to study tumor malignancy and metastasis for biological research and clinical diagnostics (Figure 12).

In recent years, polymeric nanoparticles with different architectures and formulations as drug delivery carriers based on PBA-SA recognition for cancer therapeutics were investigated by many researchers [55-57, 64]. Deshayes et al. prepared PBA-installed polymeric micelles to target sialylated epitopes overexpressed on cancer cells, and the micelles showed high affinity for SA even under intratumoral $\mathrm{pH}$ conditions, suggesting the potential 


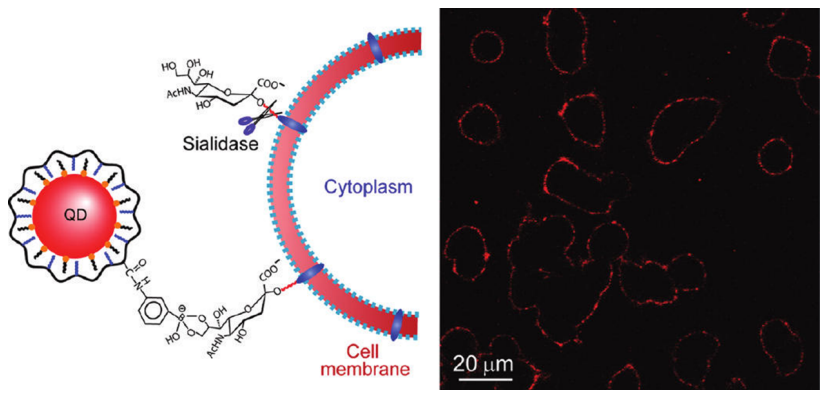

Figure 11: Specific binding of QD with SA on living cells at the C-8,9 diol of SA. Reprinted with permission from [62]. Copyright 2011, American Chemical Society

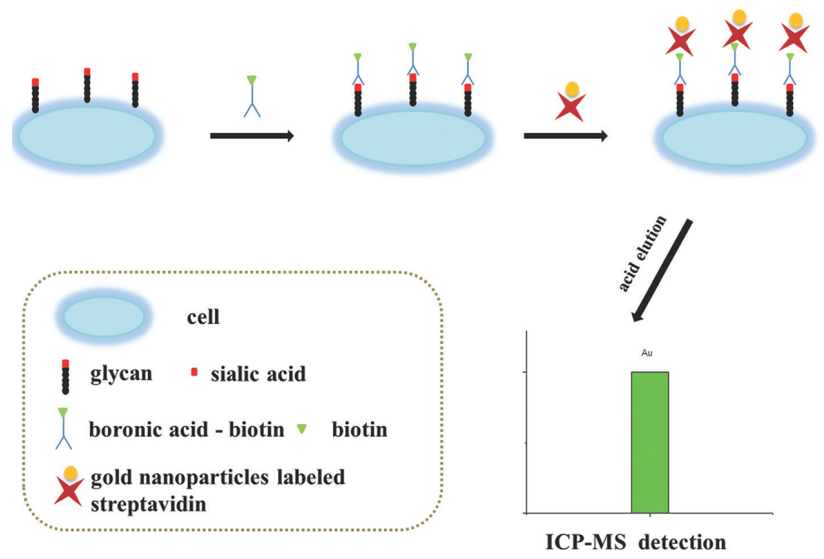

Figure 12: Illustration for recognition of SA on the cell surface assay. Reproduced with permission from [63]. Copyright 2016, The Royal Society of Chemistry

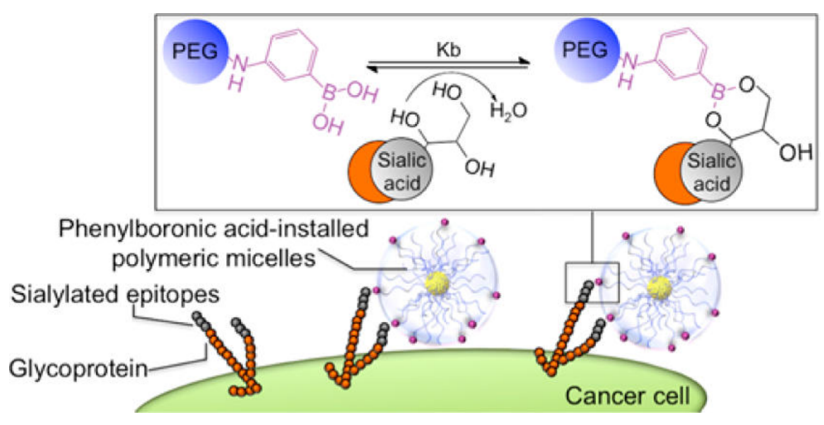

Figure 13: Preparation of PBA-installed DACHPt-loaded micelles by self-assembly of polymer-metal complex in distilled water. PBA moieties on the surface of micelles can bind to SA. Reprinted with permission from [55]. Copyright 2013, American Chemical Society

application of PBA-installed nanocarriers for enhanced tumor targeting (Figure 13) [55]. Zhao et al. developed PBA-terminated polyethylene glycol monostearate (PBAPEG- $\left.\mathrm{C}_{18}\right)$ and pluronic $\mathrm{P}_{123}\left(\mathrm{PEG}_{20}-\mathrm{PPG}_{70}-\mathrm{PEG}_{20}\right)$ targeting drug delivery system based on the selective binding with PBA [64]. Different from others' work, herein PBA located on micelles margin was protected by fructose to

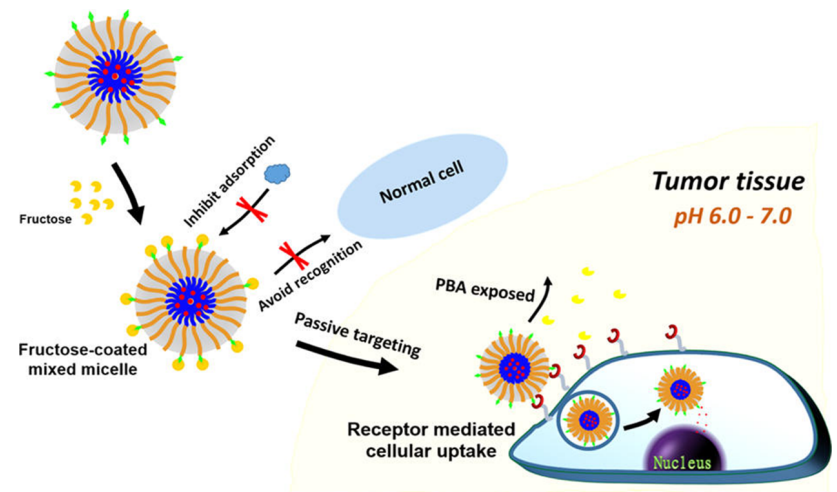

Figure 14: Illustration of $\mathrm{pH}$-activated targeting drug delivery system based on selective binding with PBA. Reprinted with permission from [64]. Copyright 2016, American Chemical Society

avoid nonspecific interaction with normal cells or other components containing cis-diol residues in vivo circulation (Figure 14).

Besides synthetic polymers, PBA could also be decorated on the natural polymers for specific targeting SA on cancer cells [65-67]. Lee et al. prepared nanoparticles based on (3-aminomethylphenyl)boronic acid (AMPB)functionalized chondroitin sulfate A (CSA)-deoxycholicacid (DOCA) to enhance tumor targeting and penetration [65]. Near-infrared fluorescence imaging revealed that CSA-DOCA-AMPB nanoparticles could target and penetrate into tumor based on both CSA-CD44 receptor and boronic acid-SA interactions. Multiple intravenous injections of DOX-loaded CSA-DOCA-AMPB nanoparticles efficiently inhibited the growth of A549 tumor in xenografted mouse models. These boronic acid-rich nanoparticles are promising candidates for cancer therapy and imaging (Figure 15). Jeong et al. fabricated AMPB-installed hyaluronic acid-ceramide (HACE)-based nanoparticles for tumortargeted delivery [66]. HACE-AMPB/MB nanoparticles improved cellular accumulation efficiency, tumor penetration efficiency, and antitumor efficacy, indicating the PBASA interaction played important roles in augmented tumor targeting and penetration (Figure 16).

PBA-based polymeric nanoparticles could also be used to deliver siRNA for cancer therapy [68, 69]. Ji et al. made PBA conjugated onto low molecular weight polyethylenimine (PEI) to generate amphiphilic PBAgrafted PEI (PEI-PBA) [68]. PEI-PBA and siRNA spontaneously self-assembled into PEI-PBA/siRNA nanocomplexes that dramatically increased siRNA uptake up to 70 - 90\% in several cancer cell lines due to the PBA-SA interaction. Moreover, the PEI-PBA nanovector effectively promoted the lysosome escape of siRNA, thereby robustly induced tumor apoptosis and cell cycle arrest. Hence, SA- 


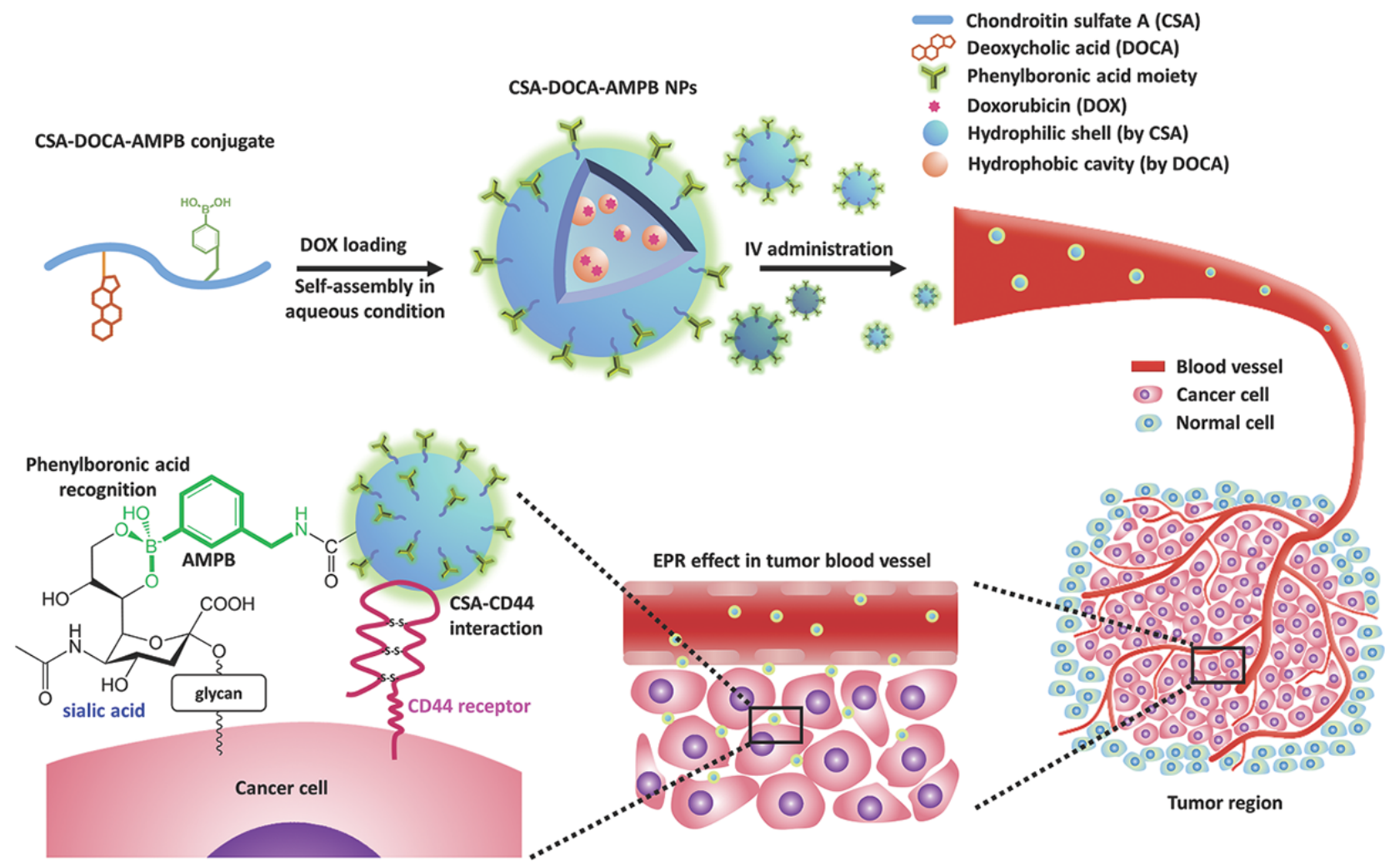

Figure 15: Schematic illustration of tumor targeting and penetrating action of CSA-DOCA-AMPB/DOX nanoparticles. Reproduced with permission from [65]. Copyright 2018, WILEY-VCH Verlag GmbH \& Co. KGaA, Weinheim

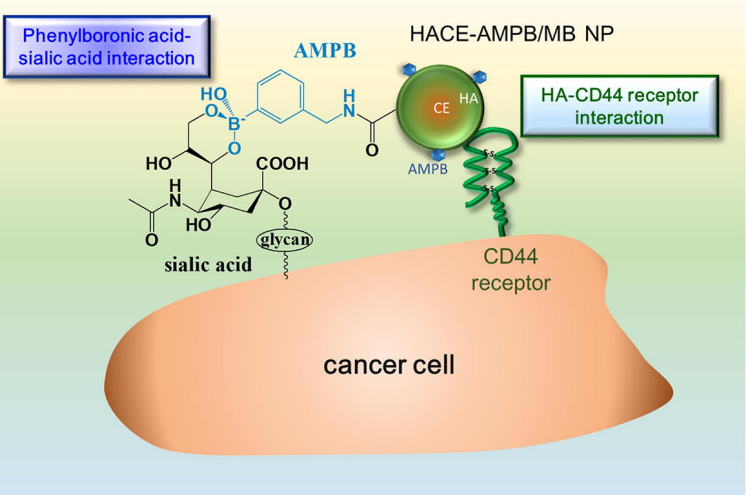

Figure 16: The tumor targeting and penetration strategy using HACEAMPB/MB nanoparticles. Reprinted with permission from [66]. Copyright 2017, Elsevier

targeted PEI-PBA could be a highly efficient and safe nanovector to improve the efficacy of cancer siRNA therapy (Figure 17). Kim et al. also reported a PBA-based polymer architecture as gene carrier for targeting anti-angiogenic tumor [69]. The nanocarrier was obtained by crosslinking of low-molecular-weight (MW) PEI to form high-MW PEI based on the interaction between PBA and galactose, re- sulting in strong interaction with anionic DNA. Inside the tumor cells, the linkages of PBA and sugar were disrupted by either acidic endosomal $\mathrm{pH}$ or intracellular ATP, resulting in effective release of preloaded gene (Figure 18). Wang et al. developed PBA-mediated chitosan nanoparticles for tumor targeting, and the results showed that PBAdecorated nanoparticles were more easily internalized by tumor cells compared to non-decorated nanoparticles in 2$\mathrm{D}$ and 3-D cell models, indicating a potential application for delivering more drugs into tumor cells due to the active targeting effect of boronic acid groups [67].

\section{Biosensor}

Due to the binding capacity of PBA and its derivatives towards free sugars or sugar-protein complexes, they have been extensively investigated as the fundamental parts of biosensor systems. The most typical sensor is glucose-sensor for detecting blood glucose concentrations in diabetes [70-75]. However, mostly of sensors were based on gold nanoparticles/surfaces owing to their plasmonic properties, PBA-based polymeric nanopartil- 
PBA-PEI

PBA-PEI/siRNA
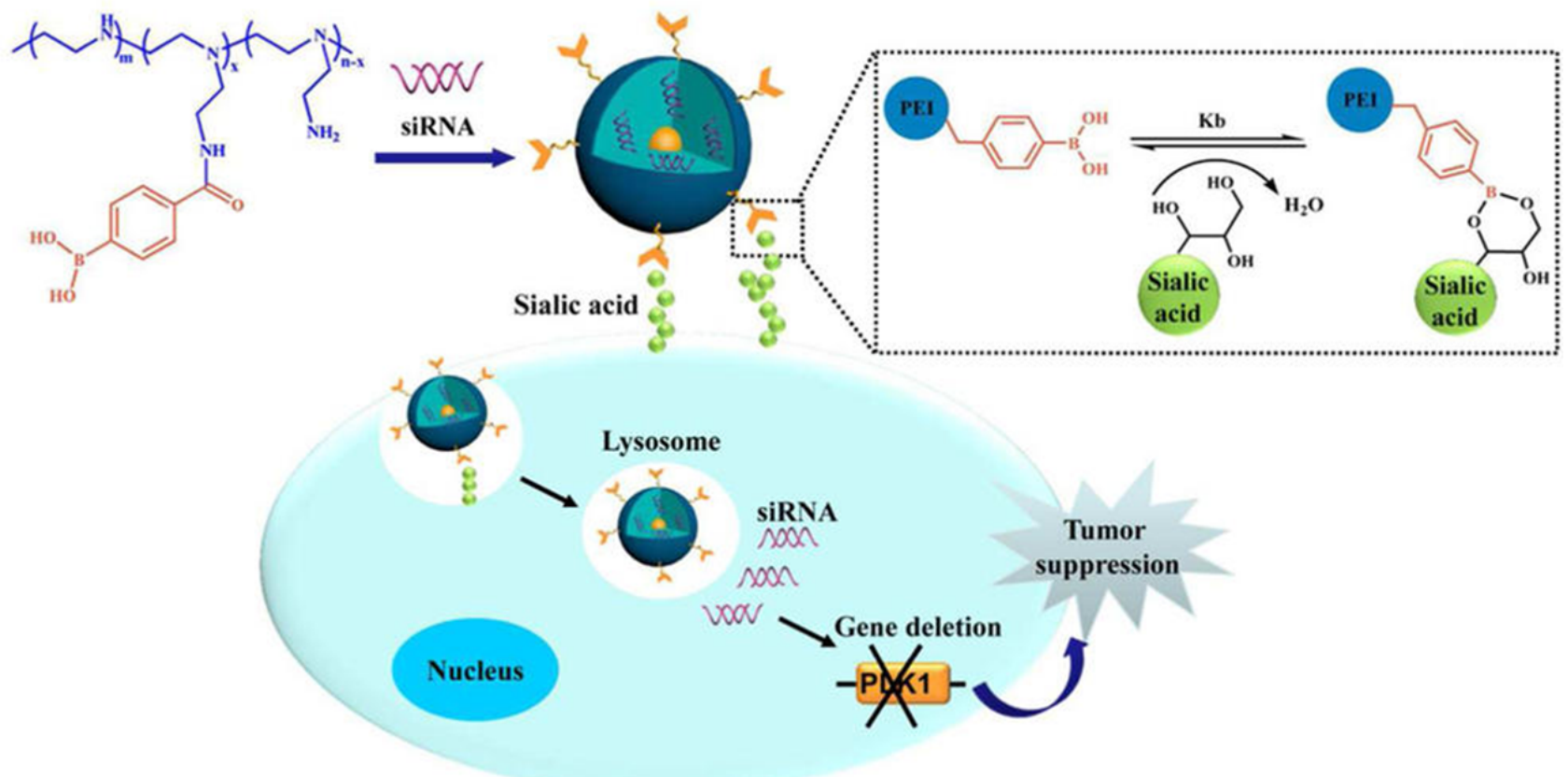

Figure 17: Synthesis of PEI-PBA conjugates for SA-targeted siRNA delivery. Reprinted with permission from [68]. Copyright 2016, American Chemical Society

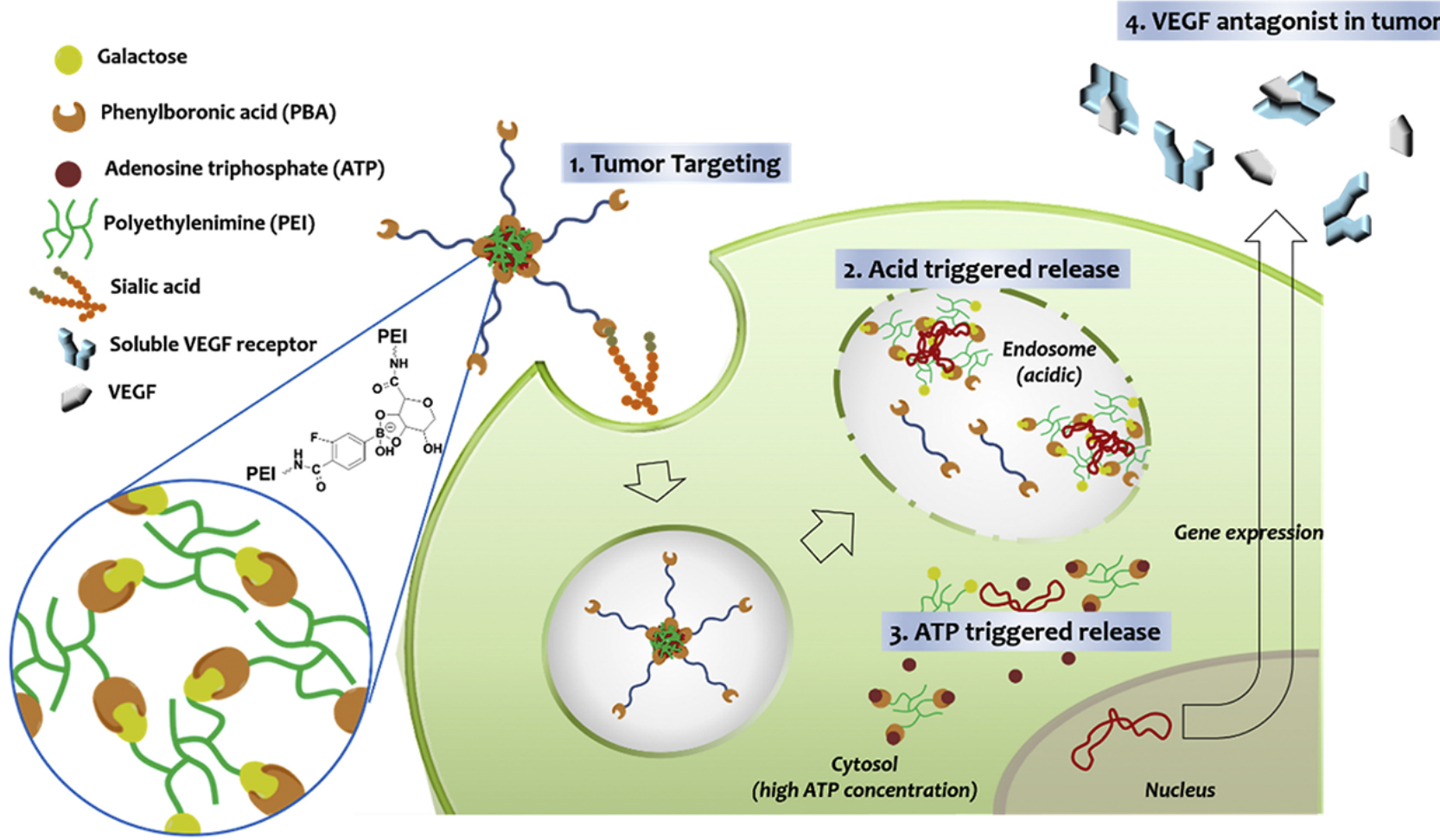

Figure 18: Schematic illustration of anti-angiogenic gene delivery mediated by PBA-PEG-Cross PEI vector. Reprinted with permission from [69]. Copyright 2015, Elsevier 


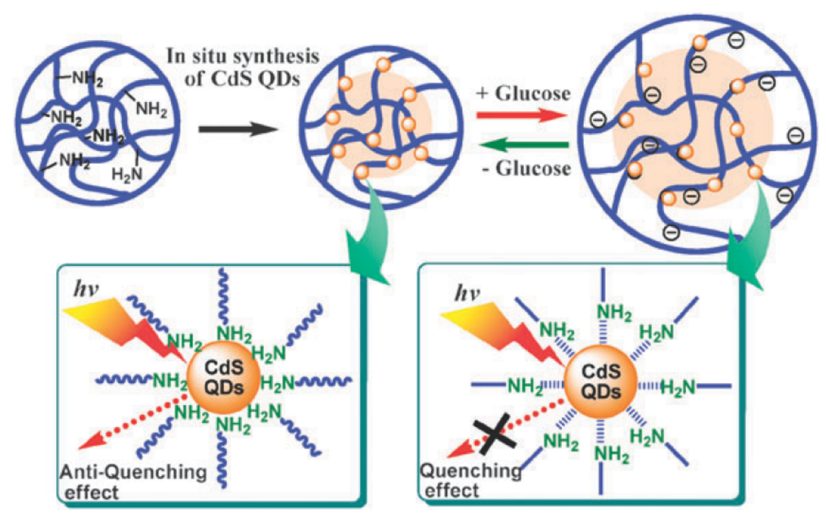

Figure 19: Reversible fluorescence quenching and anti-quenching of CdS QDs-immobilized $p$ (NIPAM-AAm-PBA) microgels in response to different glucose concentrations. Reproduced with permission from [71]. Copyright 2009, The Royal Society of Chemistry

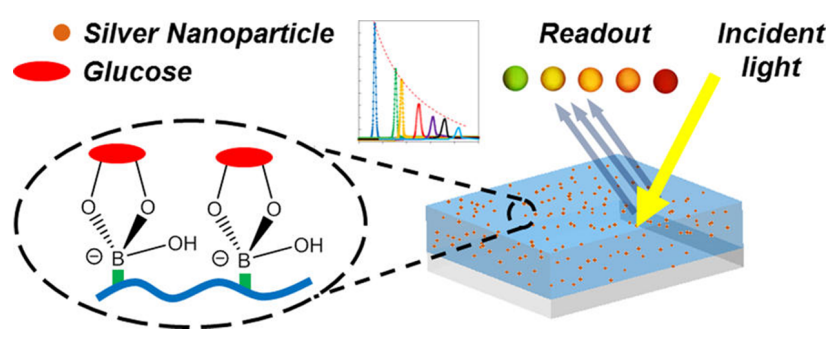

Figure 20: Illustration of the sensor for sensing the target glucose. Reprinted with permission from [74]. Copyright 2014, American Chemical Society

cles used in the nanobiosensor were seldom explored. Zhou and coworkers developed PBA-decorated CdS QDsimmobilized p(NIPAM-AAm-PBA) microgels [71]. The microgels undergone swelling and deswelling process resulting in changes in the florescence intensity of QDs in response to different glucose concentrations (Figure 19). Yetisen et al. developed a PBA-decorated PAAm matrix- $\mathrm{Ag}^{\mathrm{O}}$ nanoparticle system used as the clinically relevant optical glucose photonic nanosensor [74]. The nanosensor response was achieved within $5 \mathrm{~min}$, reset to baseline in $\sim 10$ $\mathrm{s}$, and could be reused at least 400 times without a compromise in accuracy. The sensor may have implications for reusable, equipment-free colorimetric point-of-care diagnostic devices for diabetes screening (Figure 20).

Besides detection of glucose, PBA-based sensors, though not the polymeric nanoparticle systems, could also detect SA [52, 76], glycoproteins [77, 78], bacteria [79-81] and virus $[82,83]$ based on the interaction between PBA and carbohydrates, indicating the great potential applications in the sensor fields.

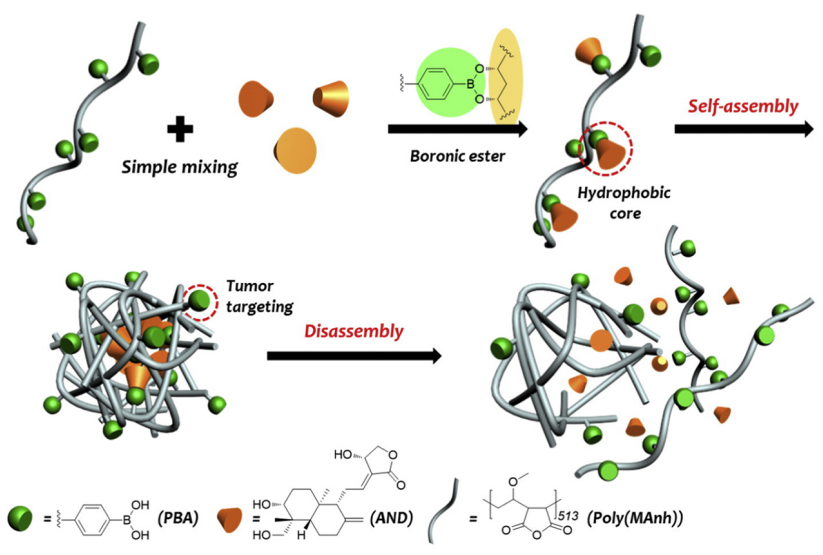

Figure 21: Schematic illustration for the formulation and destruction of pPBA-AND nanoconstruct. Reprinted with permission from [85]. Copyright 2016, Elsevier

\section{Stimuli-responsive drug delivery systems}

PBA and its derivatives can combine with cis$\mathrm{diol} /$ diphenol, and the interactions are $\mathrm{pH}$ responsive to disassemble. Some PBA derivatives are hydrogen peroxide $\left(\mathrm{H}_{2} \mathrm{O}_{2}\right)$ responsive to degrade. These properties of PBA and its derivatives could be applied in stimuli-responsive drug delivery systems.

\subsection{Diols responsiveness}

PBA can bind with a variety of cis-diols, such as galactose, mannose, glucose, 1,3-diols, etc. Their interactions are always $\mathrm{pH}$ responsive, which could be applied in the stimuliresponsive drug delivery systems [84-86]. Kim et al. formulated a water-soluble nanoconstruct by forming boronic ester between the cis-1,3-diol of andrographolide (AND) and hydrophilically polymerized phenylboronic acid (pPBA) [85]. Due to the $\mathrm{pH}$ - and diol-dependent affinity of boronic ester, AND release was regulated by low $\mathrm{pH}$ and high ATP concentrations. Besides, pPBA-AND nanoconstruct exhibited an excellent tumor targeting ability both in vitro and in vivo owing to the intrinsic property of PBA moieties (Figure 21).

\subsection{Diphenol responsiveness}

The $\mathrm{pH}$-sensitive interaction between PBA and diphenol is always used in the stimuli-responsive drug delivery systems [87-89]. Li et al. developed a DOX-loaded fluorescent nanoparticle based on the installed boronic acid- 


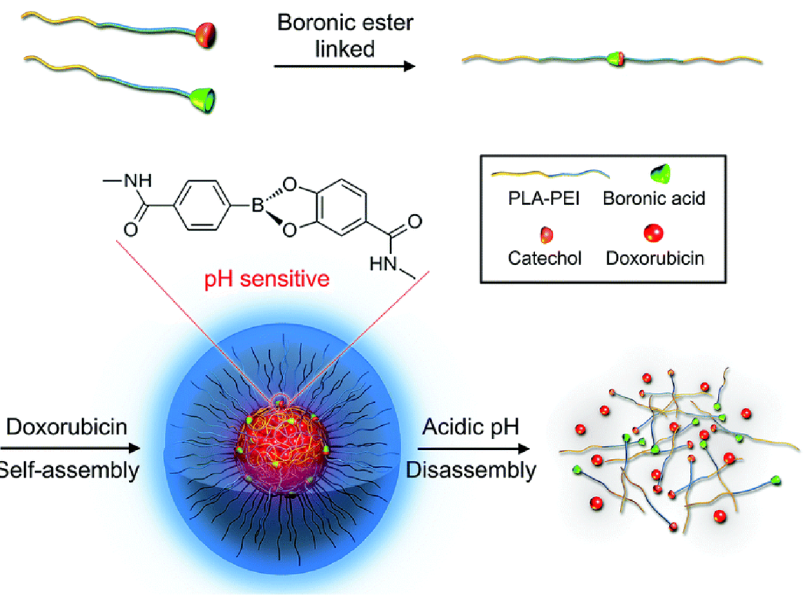

Figure 22: Schematic illustration for the formulation of FBNPsDOX nanoparticles and $\mathrm{pH}$-responsive DOX release. Reproduced with permission from [89]. Copyright 2014, The Royal Society of Chemistry

modified poly(lactic acid)-poly(ethyleneimine)(PLA-PEI) copolymers for intracellular imaging and $\mathrm{pH}$-responsive drug delivery [89]. DOX-loaded nanoparticles showed $\mathrm{pH}-$ responsive drug release and effectively suppressed the proliferation of MCF-7 cells. In addition, the fluorescent nanoparticles tracked the process of endosomal escape by real-time imaging (Figure 22).

\section{$5.3 \mathrm{H}_{2} \mathrm{O}_{2}$ responsiveness}

$\mathrm{H}_{2} \mathrm{O}_{2}$ is highly expressed in tumor site, this could be exploited in the design of $\mathrm{H}_{2} \mathrm{O}_{2}$-responsive drug delivery systems for cancer therapy. Interestingly, 4-(methylol) phenylboronic acid and its derivatives are $\mathrm{H}_{2} \mathrm{O}_{2}$-responsive to induce PBA disintegration and loaded-drug release [56, $90,91]$. Yu et al. recently designed a temperature and $\mathrm{H}_{2} \mathrm{O}_{2}$-responsive "nano-valve" of ROSP@MSN by taking advantage of mesoporous silica nanoparticles (MSN) and 4-(4,4,5,5-tetramethyl-1,3,2-dioxa borolan-2-yl)benzyl acrylate modified polymers (ROSP) [91]. ROSP@MSN could achieve cargo loading drug in cold water, and subsequently close the pore by raising temperature to obtain ROSP@MSN@DOX. Upon the stimulus of $\mathrm{H}_{2} \mathrm{O}_{2}$, ROSP@MSN@DOX exhibited excellent DOX release behavior under physiological conditions (Figure 23).

\section{Conclusion}

In this paper, some new advances of PBA-based strategies for diagnostic and therapeutic applications are re-

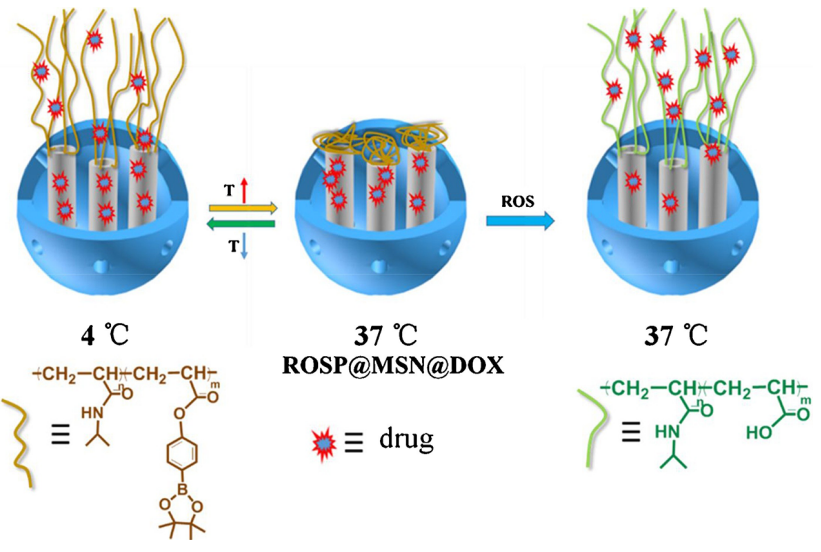

Figure 23: Schematic illustration for the temperature and $\mathrm{H}_{2} \mathrm{O}_{2}$ responsive behaviors of ROSP@MSN@DOX nanoparticles in aqueous medium. Reprinted with permission from [91]. Copyright 2017, Elsevier

viewed. Because of the versatility for different designs and the specific interactions with polyol compounds, PBA and its derivatives have been widely exploited in constructing glucose-responsive, SA-recognized and stimuliresponsive polymeric nanomaterials, such as drug delivery systems and biosensors. A series of progresses have been made in recent years including synthesis of new PBAbased polymers and fabrication of PBA-decorated nanomaterials. These PBA-decorated materials are designed to operate upon the disease microenvironment characters, such as under physiological pH or tumor acid condition. Until now, most drug delivery systems based on PBAdecorated nanoparticles were focused on insulin and anticancer drugs aiming at treatment of diabetes mellitus and cancer, respectively. And most PBA-based biosensors were focused on blood glucose detection. In conclusion, PBA and its derivatives-decorated polymers and nanomaterials have great potential applications in smart drug delivery systems and biosensors for the diagnosis and therapeutics of diseases.

Acknowledgement: This work was supported by the Guizhou Provincial Department of Education Youth Science and Technology Talent Development Project (Grant no. [2018]150), the Doctoral Foundation of Guizhou Medical University (Grant no. JY2017-28), the Science and Technology Department of Guizhou Province, Guizhou Branch of Talent Gund (Grant no. [2017]5718).

Conflict of Interests: The authors declare no conflict of interest regarding the publication of this paper. 


\section{References}

[1] Lorand J.P., Edwards J.O., Polyol complexes and structure of the benzeneboronate ion, J. Org. Chem., 1959, 24, 769-774.

[2] Van Duin M., Peters J.A., Kieboom A.P.G., Van Bekkum H., Studies on borate esters 1: The $\mathrm{pH}$ dependence of the stability of esters of boric acid and borate in aqueous medium as studied by ${ }^{11} \mathrm{~B}$ NMR, Tetrahedron, 1984, 40, 2901-2911.

[3] James T.D., Sandanayake K.R.A.S., Shinkai S., Saccharide sensing with molecular receptors based on boronic acid, Angew. Chem. Inter. Ed., 1996, 35, 1910-1922.

[4] Cambre J.N., Roy D., Sumerlin B.S., Tuning the sugar-response of boronic acid block copolymers, J. Polym. Sci., Part A: Polym. Chem., 2012, 50, 3373-3382.

[5] Vogt A.P., Trouillet V., Greiner A.M., Kaupp M., Geckle U., Barner L., Hofe T., Barner-Kowollik C., A facile route to boronic acid functional polymeric microspheres via epoxide ring opening, Macromol. Rapid Comm., 2012, 33, 1108-1113.

[6] Ting S.R.S., Chen G., Stenzel M.H., Synthesis of glycopolymers and their multivalent recognitions with lectins, Polym. Chem., 2010, 1, 1392-1412.

[7] Ma R., Shi L., Phenylboronic acid-based glucose-responsive polymeric nanoparticles: Synthesis and applications in drug delivery, Polym. Chem., 2014, 5, 1503-1518.

[8] Jin X., Zhang X., Wu Z., Teng D., Zhang X., Wang Y., Wang Z., Li C., Amphiphilic random glycopolymer based on phenylboronic acid: Synthesis, characterization, and potential as glucose-sensitive matrix, Biomacromolecules, 2009, 10, 1337-1345.

[9] Wang B., Ma R., Liu G., Liu X., Gao Y., Shen J., An Y., Shi L., Effect of coordination on the glucose-responsiveness of PEG-b-(PAA-coPAAPBA) micelles, Macromol. Rapid Comm., 2010, 31, 1628-1634.

[10] Sun L., Zhang X., Wu Z., Zheng C., Li C., Oral glucose- and pHsensitive nanocarriers for simulating insulin release in vivo, Polym. Chem., 2014, 5, 1999-2009.

[11] Kataoka K., Miyazaki H., Bunya M., Okano T., Sakurai Y., Totally synthetic polymer gels responding to external glucose concentration: Their preparation and application to on-off regulation of insulin release, J. Am. Chem. Soc., 1998, 120, 12694-12695.

[12] Hoare T., Pelton R., Engineering glucose swelling responses in poly(N-isopropylacrylamide)-based microgels, Macromolecules, 2007, 40, 670-678.

[13] Matsumoto A., Kurata T., Shiino D., Kataoka K., Swelling and shrinking kinetics of totally synthetic, glucose-responsive polymer gel bearing phenylborate derivative as a glucose-sensing moiety, Macromolecules, 2004, 37, 1502-1510.

[14] Guo H., Li H., Gao J., Zhao G., Ling L., Wang B., Guo Q., Gu Y., Li C., Phenylboronic acid-based amphiphilic glycopolymeric nanocarriers for in vivo insulin delivery, Polym. Chem., 2016, 7, 3189-3199.

[15] Kim K.T., Cornelissen J.J.L.M., Nolte R.J.M., Hest J.C.M.V.,Polymeric monosaccharide receptors responsive at neutral pH, J. Am. Chem. Soc., 2009, 131, 13908-13909.

[16] Wu W., Mitra N., Yan E.C.Y., Zhou S.,Multifunctional hybrid nanogel for integration of optical glucose sensing and selfregulated insulin release at physiological pH, ACS Nano, 2010, 4, 4831-4839.

[17] Li Y., Xiao W., Xiao K., Berti L., Luo J., Tseng H.P., Fung G., Lam K.S., Well-defined, reversible boronate crosslinked nanocarriers for targeted drug delivery in response to acidic $\mathrm{pH}$ values and cis-diols, Angew. Chem. Int. Ed., 2012, 124, 2918-2923.
[18] Matsumoto A., Ishii T., Nishida J., Matsumoto H., Kataoka K., Miyahara Y., A synthetic approach toward a self-regulated insulin delivery system, Angew. Chem. Int. Ed., 2012, 51, 2124-2128.

[19] Lee C.-S., Kim S., Kim M., Ion-sensitive field-effect transistor for biological sensing, Sensors, 2009, 9, 7111-7131.

[20] Geninatti Crich S., Alberti D., Szabo I., Aime S., Djanashvili K.,MRI visualization of melanoma cells by targeting overexpressed sialic acid with a Gd ${ }^{I I I}$-dota-en-pba imaging reporter, Angew. Chem. Int. Ed., 2013, 52, 1161-1164.

[21] Sanjoh M., Miyahara Y., Kataoka K., Matsumoto A., Phenylboronic acids-based diagnostic and therapeutic applications, Anal. Sci., 2014, 30, 111-117.

[22] Gu Z., Aimetti A.A., Wang Q., Dang T.T., Zhang Y., Veiseh O., Cheng H., Langer R.S., Anderson D.G., Injectable nano-network for glucose-mediated insulin delivery, ACS Nano, 2013, 7, 41944201.

[23] Zhao L., Xiao C., Wang L., Gai G., Ding J., Glucose-sensitive polymer nanoparticles for self-regulated drug delivery, Chem. Commun., 2016, 52, 7633-7652.

[24] Lapeyre V., Gosse I., Chevreux S., Ravaine V., Monodispersed glucose-responsive microgels operating at physiological salinity, Biomacromolecules, 2006, 7, 3356-3363.

[25] Bapat A.P., Roy D., Ray J.G., Savin D.A., Sumerlin B.S., Dynamiccovalent macromolecular stars with boronic ester linkages, J. Am. Chem. Soc., 2011, 133, 19832-19838.

[26] Roy D., Sumerlin B.S., Glucose-sensitivity of boronic acid block copolymers at physiological pH, ACS Macro Lett., 2012, 1, 529 . 532.

[27] Yao Y., Wang X., Tan T., Yang J., A facile strategy for polymers to achieve glucose-responsive behavior at neutral pH, Soft Matter, 2011, 7, 7948-7951.

[28] Kim H., Kang Y.J., Kang S., Kim K.T.,Monosaccharide-responsive release of insulin from polymersomes of polyboroxole block copolymers at neutral pH, J. Am. Chem. Soc., 2012, 134, 40304033.

[29] Kim H., Kang Y.J., Jeong E.S., Kang S., Kim K.T., Glucoseresponsive disassembly of polymersomes of sequence-specific boroxole-containing block copolymers under physiologically relevant conditions, ACS Macro Lett., 2012, 1, 1194-1198.

[30] Ma R., Yang H., Li Z., Liu G., Sun X., Liu X., An Y., Shi L., Phenylboronic acid-based complex micelles with enhanced glucoseresponsiveness at physiological $\mathrm{pH}$ by complexation with glycopolymer, Biomacromolecules, 2012, 13, 3409-3417.

[31] Liu G., Ma R., Ren J., Li Z., Zhang H., Zhang Z., An Y., Shi L., A glucose-responsive complex polymeric micelle enabling repeated on-off release and insulin protection, Soft Matter, 2013, 9, 1636-1644.

[32] Zheng C., Guo Q., Wu Z., Sun L., Zhang Z., Li C., Zhang X., Amphiphilic glycopolymer nanoparticles as vehicles for nasal delivery of peptides and proteins, Eur. J. Pharm. Sci., 2013, 49, 474-482.

[33] Guo Q., Wu Z., Zhang X., Sun L., Li C., Phenylboronate-diol crosslinked glycopolymeric nanocarriers for insulin delivery at physiological pH, Soft Matter, 2014, 10, 911-920.

[34] Cheng C., Zhang X., Wang Y., Sun L., Li C., Phenylboronic acidcontaining block copolymers: Synthesis, self-assembly, and application for intracellular delivery of proteins, New J. Chem., 2012, 36, 1413-1421.

[35] Cheng C., Zhang X., Xiang J., Wang Y., Zheng C., Lu Z., Li C., Development of novel self-assembled poly(3- 
acrylamidophenylboronic acid)/poly(2-lactobionamidoethyl methacrylate) hybrid nanoparticles for improving nasal adsorption of insulin, Soft Matter, 2012, 8, 765-773.

[36] Wang Y., Zhang X., Han Y., Cheng C., Li C., pH- and glucosesensitive glycopolymer nanoparticles based on phenylboronic acid for triggered release of insulin, Carbohydr. Polym., 2012, 89, 124-131.

[37] Guo H., Guo Q., Chu T., Zhang X., Wu Z., Yu D., Glucose-sensitive polyelectrolyte nanocapsules based on layer-by-layer technique for protein drug delivery, J. Mater. Sci. Mater. Med., 2014, 25, 121-129.

[38] Guo Q., Zhang T., An J., Wu Z., Zhao Y., Dai X., Zhang X., Li C., Block versus random amphiphilic glycopolymer nanopaticles as glucose-responsive vehicles, Biomacromolecules, 2015, 16, 3345-3356.

[39] Hoare T., Pelton R., Charge-switching, amphoteric glucoseresponsive microgels with physiological swelling activity, Biomacromolecules, 2008, 9, 733-740.

[40] Xing S., Guan Y., Zhang Y., Kinetics of glucose-induced swelling of P(NIPAM-AAPBA) microgels, Macromolecules, 2011, 44, 44794486.

[41] Zhang Y., Guan Y., Zhou S., Synthesis and volume phase transitions of glucose-sensitive microgels, Biomacromolecules, 2006, 7, 3196-3201.

[42] Wang D., Liu T., Yin J., Liu S., Stimuli-responsive fluorescent poly( $\mathrm{N}$-isopropylacrylamide) microgels labeled with phenylboronic acid moieties as multifunctional ratiometric probes for glucose and temperatures, Macromolecules, 2011, 44, 2282 2290.

[43] Tang Z., Guan Y., Zhang Y., Contraction-type glucose-sensitive microgel functionalized with a 2-substituted phenylboronic acid ligand, Polym. Chem., 2014, 5, 1782-1790.

[44] Zhao L., Xiao C., Ding J., He P., Tang Z., Pang X., Zhuang X., Chen $X$., Facile one-pot synthesis of glucose-sensitive nanogel via thiol-ene click chemistry for self-regulated drug delivery, Acta Biomater., 2013, 9, 6535-6543.

[45] Wu W., Chen S., Hu Y., Zhou S., A fluorescent responsive hybrid nanogel for closed-loop control of glucose, J. Diabetes Sci. Technol., 2012, 6, 892-901.

[46] Wu W., Shen J., Li Y., Zhu H., Banerjee P., Zhou S., Specific glucoseto-SPR signal transduction at physiological $\mathrm{pH}$ by molecularly imprinted responsive hybrid microgels, Biomaterials, 2012, 33, 7115-7125.

[47] Ye T., Jiang X., Xu W., Zhou M., Hu Y., Wu W., Tailoring the glucose-responsive volume phase transition behaviour of Ag@poly(phenylboronic acid) hybrid microgels: From monotonous swelling to monotonous shrinking upon adding glucose at physiological pH, Polym. Chem., 2014, 5, 2352-2362.

[48] Van Beek W.P., Smets L.A., Emmelot P., Increased sialic acid density in surface glycoprotein of transformed and malignant cells-a general phenomenon?, Cancer Res., 1973, 33, 2913-2922.

[49] Fuster M.M., Esko J.D., The sweet and sour of cancer: Glycans as novel therapeutic targets, Nat. Rev. Cancer, 2005, 5, 526-542.

[50] Otsuka H., Uchimura E., Koshino H., Okano T., Kataoka K., Anomalous binding profile of phenylboronic acid with $\mathrm{N}$ acetylneuraminic acid (Neu5Ac) in aqueous solution with varying pH, J. Am. Chem. Soc., 2003, 125, 3493-3502.

[51] Djanashvili K., Frullano L., Peters J.A., Molecular recognition of sialic acid end groups by phenylboronates, Chem.-Eur. J., 2005, 11, 4010-4018.
[52] Matsumoto A., Sato N., Kataoka K., Miyahara Y., Noninvasive sialic acid detection at cell membrane by using phenylboronic acid modified self-assembled monolayer gold electrode, J. Am. Chem. Soc., 2009, 131, 12022-12023.

[53] Han E., Ding L., Ju H., Highly sensitive fluorescent analysis of dynamic glycan expression on living cells using glyconanoparticles and functionalized quantum dots, Anal. Chem., 2011, 83, 7006-7012.

[54] Deng R., Yue J., Qu H., Liang L., Sun D., Zhang J., Liang C., Xu W., Xu S., Glucose-bridged silver nanoparticle assemblies for highly sensitive molecular recognition of sialic acid on cancer cells via surface-enhanced raman scattering spectroscopy, Talanta, 2018, 179, 200-206.

[55] Deshayes S., Cabral H., Ishii T., Miura Y., Kobayashi S., Yamashita T., Matsumoto A., Miyahara Y., Nishiyama N., Kataoka K., Phenylboronic acid-installed polymeric micelles for targeting sialylated epitopes in solid tumors, J. Am. Chem. Soc., 2013, 135, 1550115507.

[56] Aguirre-Chagala Y.E., Santos J.L., Huang Y., Herrera-Alonso M., Phenylboronic acid-installed polycarbonates for the $\mathrm{pH}$ dependent release of diol-containing molecules, ACS Macro Lett., 2014, 3, 1249-1253.

[57] Zhang X., Zhang Z., Su X., Cai M., Zhuo R., Zhong Z., Phenylboronic acid-functionalized polymeric micelles with a HepG2 cell targetability, Biomaterials, 2013, 34, 10296-10304.

[58] Liu H., Li Y., Sun K., Fan J., Zhang P., Meng J., Wang S., Jiang L., Dual-responsive surfaces modified with phenylboronic acidcontaining polymer brush to reversibly capture and release cancer cells, J. Am. Chem. Soc., 2013, 135, 7603-7609.

[59] Matsumura Y., Maeda H., A new concept for macromolecular therapeutics in cancer chemotherapy: Mechanism of tumoritropic accumulation of proteins and the antitumor agent smancs, Cancer Res., 1986, 46, 6387-6392.

[60] Yang Z., Sun N., Cheng R., Zhao C., Liu Z., Li X., Liu J., Tian Z., pH multistage responsive micellar system with charge-switch and PEG layer detachment for co-delivery of paclitaxel and curcumin to synergistically eliminate breast cancer stem cells, Biomaterials, 2017, 147, 53-67.

[61] Gao W., Li S., Liu Z., Sun Y., Cao W., Tong L., Cui G., Tang B., Targeting and destroying tumor vasculature with a near-infrared laser-activated "nanobomb" for efficient tumor ablation, Biomaterials, 2017, 139, 1-11.

[62] Liu A., Peng S., Soo J.C., Kuang M., Chen P., Duan H., Quantum dots with phenylboronic acid tags for specific labeling of sialic acids on living cells, Anal. Chem., 2011, 83, 1124-1130.

[63] Zhang X., Chen B., He M., Zhang Y., Peng L., Hu B., Boronic acid recognition based-gold nanoparticle-labeling strategy for the assay of sialic acid expression on cancer cell surface by inductively coupled plasma mass spectrometry, Analyst, 2016, 141, 1286-1293.

[64] Zhao D., Xu J.-Q., Yi X.-Q., Zhang Q., Cheng S.-X., Zhuo R.-X., Li F., pH-activated targeting drug delivery system based on the selective binding of phenylboronic acid, ACS Appl. Mater. Inter., 2016, 8, 14845-14854.

[65] Lee J.-Y., Chung S.-J., Cho H.-J., Kim D.-D., Phenylboronic aciddecorated chondroitin sulfate A-based theranostic nanoparticles for enhanced tumor targeting and penetration, Adv. Funct. Mater., 2015, 25, 3705-3717.

[66] Jeong J.Y., Hong E.-H., Lee S.Y., Lee J.-Y., Song J.-H., Ko S.-H., Shim J.-S., Choe S., Kim D.-D., Ko H.-J., Cho H.-J., Boronic acid-tethered 
amphiphilic hyaluronic acid derivative-based nanoassemblies for tumor targeting and penetration, Acta Biomater., 2017, 53, 414-426.

[67] Wang X., Tang H., Wang C., Zhang J., Wu W., Jiang X., Phenylboronic acid-mediated tumor targeting of chitosan nanoparticles, Theranostics, 2016, 6, 1378-1392.

[68] Ji M., Li P., Sheng N., Liu L., Pan H., Wang C., Cai L., Ma Y., Sialic acid-targeted nanovectors with phenylboronic acid-grafted polyethylenimine robustly enhance siRNA-based cancer therapy, ACS Appl. Mater. Inter., 2016, 8, 9565-9576.

[69] Kim J., Lee Y.M., Kim H., Park D., Kim J., Kim W.J., Phenylboronic acid-sugar grafted polymer architecture as a dual stimuliresponsive gene carrier for targeted anti-angiogenic tumor therapy, Biomaterials, 2016, 75, 102-111.

[70] Zenkl G., Klimant I., Fluorescent acrylamide nanoparticles for boronic acid based sugar sensing - from probes to sensors, $\mathrm{Mi}$ crochim. Acta, 2009, 166, 123-131.

[71] Wu W., Zhou T., Shen J., Zhou S., Optical detection of glucose by CdS quantum dots immobilized in smart microgels, Chem. Commun., 2009, 4390-4392.

[72] Egawa Y., Seki T., Takahashi S., Anzai J.-i., Electrochemical and optical sugar sensors based on phenylboronic acid and its derivatives, Mater. Sci. Eng. C-Mater., 2011, 31, 1257-1264.

[73] Li J., Wang Z., Li P., Zong N., Li F., A sensitive non-enzyme sensing platform for glucose based on boronic acid-diol binding, Sensors Actuat. B-Chem., 2012, 161, 832-837.

[74] Yetisen A.K., Montelongo Y., da Cruz Vasconcellos F., MartinezHurtado J.L., Neupane S., Butt H., Qasim M.M., Blyth J., Burling K., Carmody J.B., Evans M., Wilkinson T.D., Kubota L.T., Monteiro M.J., Lowe C.R., Reusable, robust, and accurate laser-generated photonic nanosensor, Nano Lett., 2014, 14, 3587-3593.

[75] Tram N., Magda J.J., Tathireddy P., Manipulation of the isoelectric point of polyampholytic smart hydrogels in order to increase the range and selectivity of continuous glucose sensors, Sensors Actuat. B-Chem., 2018, 255, 1057-1063.

[76] Zhou Y., Dong H., Liu L., Liu J., Xu M., A novel potentiometric sensor based on a poly(anilineboronic acid)/graphene modified electrode for probing sialic acid through boronic acid-diol recognition, Biosens. Bioelectron., 2014, 60, 231-236.

[77] Ang S.H., Thevarajah M., Alias Y., Khor S.M., Current aspects in hemoglobin A1c detection: A review, Clin. Chim. Acta, 2015, 439, 202-211.

[78] Wang X., Dong J., Ming H., Ai S., Sensing of glycoprotein via a biomimetic sensor based on molecularly imprinted polymers and graphene-Au nanoparticles, Analyst, 2013, 138, 1219-1225.

[79] Amin R., Elfeky S.A., Fluorescent sensor for bacterial recognition, Spectrochim. Acta A, 2013, 108, 338-341.
[80] Dechtrirat D., Gajovic-Eichelmann N., Wojcik F., Hartmann L., Bier F.F., Scheller F.W., Electrochemical displacement sensor based on ferrocene boronic acid tracer and immobilized glycan for saccharide binding proteins and E. coli, Biosens. Bioelectron., 2014, $58,1-8$.

[81] Wang J., Gao J., Liu D., Han D., Wang Z., Phenylboronic acid functionalized gold nanoparticles for highly sensitive detection of staphylococcus aureus, Nanoscale, 2012, 4, 451-454.

[82] Khanal M., Vausselin T., Barras A., Bande O., Turcheniuk K., Benazza M., Zaitsev V., Teodorescu C.M., Boukherroub R., Siriwardena A., Dubuisson J., Szunerits S., Phenylboronic-acid-modified nanoparticles: Potential antiviral therapeutics, ACS Appl. Mater. Inter, 2013, 5, 12488-12498.

[83] Huang L.-L., Jin Y.-J., Zhao D., Yu C., Hao J., Xie H.-Y., A fast and biocompatible living virus labeling method based on sialic acidphenylboronic acid recognition system, Anal. Bioanal. Chem., 2014, 406, 2687-2693.

[84] Jin Q., Lv L.-P., Liu G.-Y., Xu J.-P., Ji J., Phenylboronic acid as a sugar- and $\mathrm{pH}$-responsive trigger to tune the multiple micellization of thermo-responsive block copolymer, Polymer, 2010, 51, 3068-3074.

[85] Kim J., Lee J., Lee Y.M., Pramanick S., Im S., Kim W.J., Andrographolide-loaded polymerized phenylboronic acid nanoconstruct for stimuli-responsive chemotherapy, J. Control. Release, 2017, 259, 203-211.

[86] Prosperi-Porta G., Kedzior S., Muirhead B., Sheardown H., Phenylboronic-acid-based polymeric micelles for mucoadhesive anterior segment ocular drug delivery, Biomacromolecules, 2016, 17, 1449-1457.

[87] Ren J., Zhang Y., Zhang J., Gao H., Liu G., Ma R., An Y., Kong D., Shi L., $\mathrm{pH} /$ sugar dual responsive core-cross-linked PIC micelles for enhanced intracellular protein delivery, Biomacromolecules, 2013, 14, 3434-3443.

[88] Jia H.-z., Zhu J.-y., Wang X.-l., Cheng H., Chen G., Zhao Y.-f., Zeng X., Feng J., Zhang X.-Z., Zhuo R.-x., A boronate-linked linearhyperbranched polymeric nanovehicle for $\mathrm{pH}$-dependent tumortargeted drug delivery, Biomaterials, 2014, 35, 5240-5249.

[89] Li S., Hu K., Cao W., Sun Y., Sheng W., Li F., Wu Y., Liang X.-J., $\mathrm{pH}$-responsive biocompatible fluorescent polymer nanoparticles based on phenylboronic acid for intracellular imaging and drug delivery, Nanoscale, 2014, 6, 13701-13709.

[90] Aguirre-Chagala Y.E., Santos J.L., Aguilar-Castillo B.A., HerreraAlonso M., Synthesis of copolymers from phenylboronic acidinstalled cyclic carbonates, ACS Macro Lett., 2014, 3, 353-358.

[91] Yu F., Wu H., Tang Y., Xu Y., Qian X., Zhu W., Temperature-sensitive copolymer-coated fluorescent mesoporous silica nanoparticles as a reactive oxygen species activated drug delivery system, Int. J. Pharm., 2017, 536, 11-20. 